\title{
D-08
}

\section{INFLUENCIA DE LA EXPROPIACIÓN FORZOSA EN LA DISPONIBILIDAD DE TERRENOS PARA LA EJECUCIÓN DE OBRAS DE MODERNIZACIÓN DE REGADÍOS}

\author{
Martín de la Torre Martínez, C (1) (P); Serrano Bermejo, A (2);De la Fuente García, E (3)
}

${ }^{1}$ Responsable Unidad de Expropiaciones, Gerencia de Ingeniería y Edificación (Tragsatec), Julián Camarillo 6B, 28037, Madrid, cmam@tragsa.es

2 Profesor Titular, Universidad Politécnica de Madrid, Escuela Técnica Superior de Ingenieros Agrónomos, Departamento de Economía Agraria, Estadística y Gestión de Empresas, Av. Puerta de Hierro 2, 28040, Madrid, arturo.serrano@upm.es

${ }^{3}$ Jefe de Actuaciones y Proyectos, Gerencia de Ingeniería y Edificación (Tragsatec), Julián Camarillo 6B, 28037, Madrid, emfg@tragsa.es

\section{Resumen}

La evolución de la economía del país, ha hecho que los tipos de interés hayan disminuido de manera continuada en los últimos años. El hecho de que la determinación del valor de expropiación desde el año 2.007 se lleve a cabo mediante capitalización de rentas y que, por lo tanto, el valor de indemnización dependa del tipo de interés, ha ocasionado un efecto económico difícil para las partes beneficiarias en una expropiación.

En este trabajo se estudia la evolución de la normativa, se cuantifican los resultados para el caso de una finca concreta de una explotación de algodón de regadío en el valle del Guadalquivir y se analizan los resultados.

En el estudio puede observarse que en los últimos ocho años, el valor de mercado de la tierra ha seguido una tendencia a la baja. Por el contrario, el valor de indemnización por expropiación de la misma se ha disparado, lo que hace inviable a las comunidades de regantes la participación en la modernización de regadíos, así como a la Administración la aplicación del Plan Hidrológico Nacional o Plan Nacional de Regadíos.

Estas circunstancias han hecho que, en octubre de 2.015 (Ley de carreteras) y noviembre de 2.015 (Texto refundido de Ley de Suelo), la legislación haya intentado paliar la situación, aunque sigue siendo insuficiente. Por ello se proponen algunas medidas a modo de conclusión al final del trabajo.

\begin{abstract}
The evolution of the country's economy has made that interest rates have decreased continuously in recent years. The fact that since the year 2007 the determination of the value of expropriation is being carried out through revenue capitalization, in which the indemnification value depends on the interest rate, has caused a difficult economic impact in the recipient parties of an expropriation.
\end{abstract}

In this paper we study the evolution of the expropriation regulations as well as the assessment of the amount of money corresponding to the expropriation of a farm dedicated the production of cotton in the Guadalquivir valley.

In the study it can be seen that over the last eight years the market value of the land has followed a downward trend. On the other hand, the value of compensation for land 
expropriation has been triggered, making infeasible to the irrigation communities their participation in the modernization of the irrigation systems and hinders the Administration the implementation of the National Hydrological Plan or the National Irrigation Plan.

These circumstances have led the Government to try to alleviate the situation with new regulations (road act of October 2015 and revised text of law of soil of November 2015) which remain insufficient. Therefore some legislative proposals are given as conclusions at the end of this work.

\section{1 - Introducción. Necesidad de Expropiación. Requisitos Previos.}

Con la modernización de regadíos, España promueve dirigir la agricultura hacia la competitividad, rentabilidad y sobre todo a la mejora de la eficiencia del uso del agua de riego. Supone un impulso positivo en la gestión y uso razonable del agua, que repercute directamente en la productividad de las explotaciones, y un valor añadido bruto de la zona modernizada.

De esta forma, los poderes públicos han acometido una planificación importante de reorientación para la mejora, la consolidación y la modernización de los regadíos españoles, que se recogen, entre otros, en el Plan Hidrológico Nacional, Plan Nacional de Regadíos, y en los distintos Planes de Choque para la Mejora y Modernización de los Regadíos.

El punto de partida necesario e ineludible para conseguir esta Modernización, radica en la obtención de la disponibilidad de los terrenos necesarios sobre los que se proyectan las actuaciones de mejora. Esta disponibilidad se encuentra ligada directamente al consentimiento o autorización de aquellas personas físicas o jurídicas que ostentan la titularidad de estos terrenos. Por tanto, la voluntad de los particulares puede incidir en la ejecución del planteamiento de modernización y, consecuentemente, subjetivizar el desarrollo de las obras.

Para evitar esta arbitrariedad en la obtención de la disposición de los terrenos necesarios, y conseguirlos de forma rápida y efectiva es conveniente iniciar por parte de la Administración competente el procedimiento expropiatorio correspondiente, mediante el cual se resuelve la confrontación existente entre el interés particular del expropiado y el interés general que conlleva la actuación, haciendo prevalecer éste último sobre la voluntad de los particulares, los que deberán de ser resarcidos o compensados siempre a cambio de una indemnización sustitutoria del bien o derecho expropiado a través de la figura del justoprecio.

El mecanismo legal por el que se desarrolla el procedimiento expropiatorio se encuentra en la Ley de Expropiación Forzosa, de 16 de diciembre de 1954, y en su Reglamento aprobado y desarrollado por Decreto de 26 de abril de 1957. Esta potestad expropiatoria sólo puede ser ejercida si existe una causa de utilidad pública o interés social que la legitime. Así se recoge en nuestra Carta Magna, en su artículo 33.3 cuando establece que "Nadie podrá ser privado de sus bienes y derechos sino por causa justificada de utilidad pública o interés social, mediante la correspondiente indemnización y de conformidad con lo dispuesto por las leyes". Por ello, la declaración de la causa que motiva la expropiación es indispensable para proceder a cualquier expropiación.

Por tanto, el objetivo principal de la incoación de un expediente expropiatorio es la obtención de la disponibilidad de los terrenos necesarios para la ejecución de obras de una forma rápida y efectiva. En la citada Ley de Expropiación Forzosa y su Reglamento se contemplan y desarrollan dos tipos de procedimientos en los expedientes de expropiación forzosa: el ordinario y el excepcional o de urgente ocupación, siendo éste último, el más 
usualmente aplicado por su rápida tramitación en la obtención y disposición de terrenos y, por tanto, en el que nos centramos en la presente exposición.

Para que la disponibilidad de los terrenos se haga efectiva mediante la tramitación de un expediente expropiatorio por la vía de urgencia, conforme establece el artículo 52 de la Ley de Expropiación forzosa, es necesario que las obras a ejecutar cumplan previamente con una serie de requisitos como son:

a) Que se encuentren declaradas de interés general, de utilidad pública y de urgente ocupación.

b) La existencia de un proyecto técnico, que contemple las obras a realizar, debidamente aprobado por la Administración competente, y que contenga un Anejo de expropiaciones donde se relacione de forma detallada todos los bienes y derechos necesarios para la ejecución de la obra.

Finalmente, en cuanto a la competencia para acordar la expropiación y los beneficiarios de la misma, la vigente Ley de Expropiación de 1954, establece en su artículo 2 que la expropiación puede ser acordada por el Estado, la provincia (actualmente la Comunidad Autónoma) y el municipio, pero amplía la causa de interés social de la expropiación a los particulares, en los que concurran los requisitos señalados por la Ley especial necesaria a estos efectos, que podrán ser beneficiarios de la expropiación. En el caso que nos ocupa, la expropiación tiene como beneficiario final a las comunidades de regantes, que pueden ostentar esta condición por su carácter de entidades de derecho público.

\section{2 - Expediente de Expropiación en Obras de Modernización de Regadíos.}

Como ya hemos comentado, en el contexto de obras de modernización de regadíos, el expediente expropiatorio se suele tramitar por el procedimiento de urgencia, regulado en el artículo 52 de la Ley de Expropiación Forzosa. Este procedimiento "excepcional", abrevia de manera sustancial la disponibilidad de los terrenos respecto al procedimiento ordinario, ya que posibilita que primeramente se efectúe la ocupación del bien expropiado y posteriormente se procede a tramitar la determinación del justiprecio, invirtiendo así el orden de actuación respecto al procedimiento ordinario. Es por ello, que este procedimiento de urgencia, ha ido convirtiéndose en la forma sistemática y generalizada del expediente expropiatorio por su mayor agilidad, rapidez y eficacia en los fines pretendidos, dejando en la práctica extinto el procedimiento ordinario, ya que en éste la ocupación del bien queda diferida a la entrega del justiprecio al expropiado.

Como resumen de la tramitación del expediente expropiatorio por el procedimiento de urgencia, se puede establecer el siguiente orden:

1. Declaración de interés general, de utilidad pública y declaración de urgente necesidad de ocupación.

2. Inicio y tramitación del expediente expropiatorio conforme al procedimiento establecido en el artículo 52 de la Ley de Expropiación Forzosa.

3. Ocupación y toma de posesión por la Administración de los terrenos expropiados.

4. Determinación del justiprecio.

5. Pago del bien o derecho expropiado

En lo referente a las obras de modernización de regadíos relacionadas tanto con el Plan Nacional de Regadíos como con el Plan de Choque, todas ellas han sido declaradas de interés general. Dichas obras llevan implícitas las declaraciones de utilidad pública a los 
efectos previstos en los artículos 9, 10 y 11 de la Ley de 16 de diciembre de 1954 de Expropiación Forzosa, y la de urgencia, a los efectos de ocupación de los bienes afectados a los que se refiere el art. 52 de dicha ley.

En relación al tipo de ocupación y privación del dominio de la propiedad, pueden establecerse tres tipos de afecciones: expropiación del pleno dominio, imposición de servidumbres, que suponen una limitación en el dominio o uso y disfrute de la cosa expropiada por parte de la propiedad, y ocupaciones temporales de los terrenos durante la duración de las obras.

Las grandes infraestructuras en obras de regadíos como la ejecución de balsas de riego, canales, estaciones de bombeo, filtrado y arquetas, entre otras, significan la privación total al titular del dominio del terreno ocupado por las mismas y, por tanto, su expropiación definitiva. Las conducciones de tubería, o tendidos eléctricos conllevan la imposición de servidumbres de acueducto, en el primer caso, y de vuelo, en el segundo, que suponen para el titular una limitación a su dominio pero no la pérdida total del mismo. Por último, la ocupación temporal de los terrenos necesarios durante el tiempo que dure la ejecución de la obra al objeto del tránsito de maquinaria, personal y zonas de acopio para la obra.

Conforme establece la Ley de Expropiación Forzosa y su Reglamento, en la valoración del justiprecio, se deberá valorar cada una de estas afecciones y siempre en directa relación a su extensión, cultivo, estado de la parcela, etc. Además habrá de tenerse en cuenta otro tipo de partidas indemnizatorias, complementarias a las principales afecciones, y que se concretan en los posibles deméritos ocasionados por restos de finca sin expropiar dejando una superficie que resulta antieconómica su explotación, o los derivados de la partición de finca, que suponen una anulación de la unidad de explotación, como también es necesario que se contemple la indemnización por rápida ocupación y el premio de afección.

Finalmente la Ley establece que a este justiprecio se puede llegar bien mediante acuerdo entre las partes, y en ausencia de este, establece un mecanismo de actuación hasta llegar a su fijación por el Jurado Provincial de Expropiación Forzosa.

\section{3 - Criterios para evaluar el impacto de la expropiación forzosa}

De lo comentado anteriormente puede deducirse que en obras importantes de modernización de regadíos, los costes derivados de la disponibilidad de terrenos tienen una significación importante dentro del cómputo total de la inversión a realizar. Requiere un análisis profundo tanto del trámite administrativo como del régimen jurídico de valoración de los terrenos que resulta de aplicación a la hora de fijar el justiprecio, un correcto dimensionamiento de las afecciones que concurren y una estimación del valor de los terrenos expropiados a nivel de anejo de expropiaciones del proyecto lo suficientemente aproximada al valor real de la totalidad de las afecciones y su posible variación a lo largo de la vida la tramitación del expediente expropiatorio.

El impacto económico de la expropiación refiere unívocamente a la fijación del precio unitario del suelo, en la instancia que corresponda, en tanto en cuanto todas las afecciones soportadas por el terreno tienen su origen estimativo en este valor. Así, cuando se determina el valor unitario de suelo, este será la referencia para valorar la expropiación permanente, la servidumbre, ocupaciones temporales, indemnización por rápida ocupación, deméritos y premio de afección.

A continuación se detalla con mayor profundidad aquellos aspectos legales y técnicos que influyen de una manera notoria en la fijación de este valor unitario de suelo en un procedimiento expropiatorio. 


\subsection{Desarrollo Normativo de Valoración}

La elección del método valorativo de una finca rústica viene determinada por la finalidad misma de la valoración. De este modo, el criterio a adoptar varía si se trata de una compraventa rústica o tiene una finalidad hipotecaria o expropiatoria.

Si bien la regulación expropiatoria atiende de manera general a la tasación de los terrenos expropiados, desde la Ley, de 12 de mayo de 1956, sobre régimen del suelo y ordenación urbana, la legislación del suelo ha venido conformando un régimen excepcional de valoración desplazando el criterio general contenido en la LEF y su Reglamento.

Para tener una idea ajustada a la actualidad valorativa a efectos expropiatorios, conviene destacar que desde el año 2007, fecha en la que entra en vigor La Ley 8/2007, de 28 de mayo, de suelo, se han sucedido numerosos cambios legislativos y normativos que han afectado principalmente y de manera significativa al criterio de valoración a adoptar para la fijación de los justiprecio. De este, como resumen del recorrido legislativo, se pueden citar:

- $\quad$ La Ley 8/2007, de 28 de mayo, de suelo

- Real Decreto Legislativo 2/2008, de 20 de junio, por el que se aprueba el texto refundido de la Ley de suelo

- Real Decreto 1492/2011, de 24 de octubre, por el que se aprueba el Reglamento de valoraciones de la Ley de Suelo

- $\quad$ Ley $37 / 2015$, de 29 de septiembre, de carreteras

- Real Decreto Legislativo 7/2015, de 30 de octubre, por el que se aprueba el texto refundido de la Ley de Suelo y Rehabilitación Urbana.

La Ley $8 / 2007$, de 28 de mayo, de suelo, hoy derogada pero tuvo su vigencia hasta el 27 de junio de 2008, y la aprobación de su texto refundido en el Real Decreto Legislativo 2/2008 y la vigencia actual del Real Decreto Legislativo 7/2015 suponen un cambio definitivo tanto en la clasificación del suelo como en el criterio a adoptar para realizar las valoraciones de las afecciones a fincas rústicas en los procesos expropiatorios con respecto a la Ley 6/1998, de 13 de abril, sobre régimen del suelo y valoraciones, consolidando como método de valoración la capitalización de renta real o potencial.

A continuación, se desarrollan los aspectos fundamentales que inciden de una manera directa en la fijación del precio del suelo:

\subsubsection{Clasificación del suelo}

La Ley 6/1998 establecía tres clases de suelo atendiendo a su grado de transformación: urbano, no urbanizable y urbanizable. Se entiende por suelo urbano aquél que ya se encuentra totalmente transformado o en ejecución de un planeamiento y el suelo no urbanizable como aquel suelo en el que concurren situaciones concreta que impiden su transformación urbano, bien por la preservación de valores agrarios, forestales, ganaderos o aprovechamientos de riquezas naturales o bien por la inadecuación del mismo a ser urbano por condicionantes paisajísticos o regímenes especiales de protección. La Ley deja como suelo urbanizable aquél que no cumple ni con las condiciones de urbano ni no urbanizable.

La ley 8/2007, y siguientes desarrollos normativos lo mantienen, simplifica concepción de suelo y reduce el número de clase a dos: urbanizado y rural.

Define como suelo urbanizado aquél que se encuentra integrado de forma legal y efectiva en la red de dotaciones y servicios propios de los núcleos de población. Es decir, 
las parcelas, estén o no edificadas, que cuenten con las dotaciones y los servicios requeridos por la legislación urbanística o puedan llegar a contar con ellos sin otras obras que las de conexión de las parcelas a las instalaciones ya en funcionamiento.

\subsubsection{Método de valoración de finca en suelo rural}

Si bien la anterior norma jurídica, la Ley 6/1998, de 13 de abril, sobre régimen del suelo y valoraciones, establecía como criterio principal de valoración del suelo no urbanizable, el método de comparación a partir de fincas análogas, la ley del suelo de 2007 establece un criterio analítico de valoración en fincas ubicadas en suelo rural aplicando el método de capitalización de la renta anual o potencial, atendiendo al uso, disfrute o explotación. Es decir, es la capitalización de la renta generada por una explotación en el medio rural, entendida esta como un coste de oportunidad a un determinado interés la que genera el valor del suelo, en contraposición a la determinación del valor del suelo en base a criterios comparativos de fincas similares, en el espacio o en el tiempo, basados en los datos obtenidos de transmisiones de bienes inmuebles rústicos, principalmente operaciones de compraventa que se realizan en un determinada zona, término municipal o paraje.

La principal motivación que lleva al legislador a cambiar el criterio de valoración es el intento de eliminar o minimizar los fallos del mercado y las tensiones especulativas, atendiendo a un principio de tasación de la realidad y no estimación de las expectativas del suelo, cumpliendo, por tanto, con lo establecido en la LEF que determina que las tasaciones expropiatorias no han de tener en cuenta las plusvalías derivadas de las obras a acometer ni las previsibles para el futuro. Debe determinarse el valor del suelo atendiendo a lo que hay, independientemente de su clasificación futura y, para ello, la renta territorial se convierte en el factor principal en la formación del precio de la tierra.

Este cambio de criterio no está exento de controversia. El método de comparación, sumergido de lleno en las expectativas y en las tensiones concretas del mercado, atiende de una manera objetiva a factores que generan valor al suelo en una determinada zona o región, como pueden ser localización específica del paraje, climatología, factores edáficos concretos, tendencia futura de precios, etc... si la disponibilidad de datos para su cálculo es fiable, concreta y de calidad, que no siempre pueden ser dimensionados en el método de capitalización debido a que se corresponden con factores intangibles o en expectativas generadas, como puede ser la urbanística, no imputables a una explotación agraria. Sin embargo, el método de capitalización de rentas, en un principio atiende a la potencialidad productiva como factor exclusivo de generación del valor, aunque como se verá posteriormente, se introduzcan parámetros que intentan adaptar este valor a su entorno socio-económico desarrollados reglamentariamente. Por lo tanto, con este método, puede generalizarse que a mayor renta arrojada por la explotación mayor precio del suelo.

Se puede concluir pues, que el método comparativo tiene como pretensión estimar el valor de mercado con la dinámica de precios del mismo, atendiendo principalmente al destino del suelo y el método analítico atiende principalmente a la situación actual y real del suelo en base a la actividad productiva como generadora de su valor real y objetivo.

\subsubsection{El método de capitalización de rentas y su desarrollo reglamentario}

A grandes rasgos, el método analítico consiste en la capitalización de la renta de la tierra a un tipo de interés fijado legalmente.

La ley 8/2007 y el RD 2/2008 establece que "los terrenos se tasarán mediante la capitalización de la renta anual real o potencial, la que sea superior, de la explotación según su estado en el momento al que deba entenderse referida la valoración". 
Se define renta anual, potencial o real, como la diferencia entre ingresos y gastos anuales atendiendo al rendimiento del uso, disfrute o explotación de que sean susceptibles los terrenos conforme a la legislación que les sea aplicable. Este rendimiento del uso puede ser calculado a partir de las cuentas reales de una explotación en concreto o a partir de datos estadísticos significativos de una determinada zona, atendiendo a las prácticas comúnmente desarrolladas conformado el concepto de renta potencial.

Esta renta se capitaliza a un tipo de interés fijado inicialmente como la última referencia publicada por el Banco de España del rendimiento de la deuda pública del Estado en mercados secundarios a tres años corregido posteriormente, vía Ley de Presupuestos generales del Estado, al rendimiento de la deuda pública del Estado en mercados secundarios de dos a seis años, es decir, letras y bonos del Estado de dos a seis años.

Además, se incorpora el factor localización al valor obtenido por capitalización se podrá corregir al alza hasta un máximo del doble en función de factores objetivos de localización, como la accesibilidad a núcleos de población o a centros de actividad económica o la ubicación en entornos de singular valor ambiental o paisajístico.

Con la aprobación en el año 2011 del reglamento de Valoraciones definido en el Real Decreto 1492/2011, de 24 de octubre, por el que se aprueba el Reglamento de valoraciones de la Ley de Suelo se fija el método de cálculo de varios factores decisivos en el cálculo del valor del suelo que se habían quedado descritos pero no concretados. De este modo, como primera adecuación normativa del método, se establecen las fórmulas de cálculo de capitalización de la renta de explotación que con carácter general, en explotaciones constantes a lo largo del tiempo, se corresponde con la siguiente expresión:

$$
V=F . L \times R / r
$$

Siendo R la renta de explotación, $r$ el tipo de capitalización a aplicar y F.L el factor global de localización.

Sobre el tipo de capitalización a aplicar, fijándonos exclusivamente en explotaciones agropecuarias, el Real Decreto 1492/2011 establece:

a) Como tipo de capitalización aplicable con carácter general, $r 1$, se utilizará el establecido en el apartado 1 de la Disposición adicional séptima del texto refundido de la Ley de Suelo, es decir el rendimiento de las letras y bonos del Tesoro de dos a seis años.

b) Cuando en el suelo rural se desarrollen actividades agropecuarias o forestales, se utilizará como tipo de capitalización, r2, el resultado de multiplicar el tipo de capitalización general $\mathrm{r} 1$ por el coeficiente corrector establecido en la tabla del Anexo I de este Reglamento según el tipo de cultivo o aprovechamiento.

Cabe mencionar la importancia del factor global de localización puesto que su aplicación ha sido motivo de controversia. El reglamento de valoraciones fija el criterio para su cálculo atendiendo a la localización, impacto demográfico y socio-económico y paisajístico y medioambiental, fijando su valor como máximo en 2 respetando así lo establecido en RD 2/2008. Esta limitación en la corrección por localización se declara inconstitucional y nula por Sentencia del TC 141/2014, de 11 de septiembre, y ya el RD 7/2015 elimina la limitación superior de este factor.

El actual y vigente RD 7/2015, que aprueba el nuevo texto refundido de la ley del suelo, mantiene el criterio de valoración recogido en el Reglamento de Valoraciones pero introduce algunas modificaciones. Además de la ya comentada sobre el valor máximo del 
factor global de localización, introduce una modificación sobre el tipo de capitalización, en un intento por eliminar las distorsiones ocasionadas con el texto de la legislación anterior.

Por lo tanto, para fijar el valor unitario de suelo, es necesario estimar de una manera real o potencial la renta de explotación y aplicar el tipo de capitalización legal y el factor global de localización.

\subsubsection{Renta de la tierra}

Una vez analizado el método de valoración, es importante determinar el concepto de renta de la tierra en el planteamiento del método de capitalización. De una forma general, la renta de la tierra puede entenderse de dos formas: como el balance que arroja una finca teniendo en cuenta sus ingresos y gastos o como el canon de arrendamiento que se paga o que podría pagarse por el uso de la tierra en caso de arrendamiento.

De este modo se contempla el concepto de renta en el reglamento de valoraciones, pero no como territorial sino como renta de explotación y además, matiza el concepto de renta real y renta potencial. Por renta real se considera aquella que corresponde a la explotación del suelo rural de acuerdo con su estado y actividad en el momento de la valoración, ya sea la existente debidamente acreditada o la atribuible, de acuerdo con los cultivos y aprovechamientos implantados sobre la base de datos estadísticamente significativos. Por renta potencial se entiende aquella que pueda ser atribuible a la explotación de acuerdo con los usos y actividades más probables de que sean susceptibles, utilizando los medios técnicos normales para su producción. Los usos y actividades a los que se refiere la definición deben ser acreditados, bien porque haya una muestra estadística significativa de usos en su ámbito geográfico, es decir, que realmente se pueda destinar la explotación de la finca a cultivos y aprovechamientos comunes en la zona o porque se disponga de estudios económicos que justifiquen la viabilidad de la explotación que se quiere llevar a cabo.

Pero en un procedimiento expropiatorio, cuando se analiza y estudia la zona que va a ser afectada y se estiman los precios probables que la administración o la entidad beneficiaria va a proponer como justiprecio, no se dispone de los datos concretos de las explotaciones afectadas, de su análisis de ingresos y costes, ni de su particular "hacer" en su entorno productivo. De este modo y atendiendo a la norma, la manera de fijar la renta de explotación se realiza a partir de la información técnica y económica procedente de estudios y publicaciones realizadas por las Administraciones Públicas competentes en la materia sobre rendimientos, precios y costes, así como de las demás variables técnico-económicas de la zona. El propietario afectado sí posee información concreta de su explotación y, en caso de no estar de acuerdo con el precio ofrecido, podrá acreditar éstos en su hoja de aprecio y fundamentar de una manera robusta su valoración.

Por este motivo cobra especial importancia la información que las distintas Administraciones puedan suministrar de zonas de cultivo, cuanto más acotadas geográfica y técnicamente mejor, puesto que este aspecto redunda en una justa valoración del bien o derecho afectado. De hecho, cuando se analizan Resoluciones de justiprecio de los distintos Jurados de Expropiación, no existe un criterio unificado a la hora de distribuir ingresos y gastos para explotaciones análogas y tampoco una justificación motivada sobre el valor que se alcanza.

\subsubsection{Tipo de capitalización}

El tipo de capitalización a aplicar para valorar las fincas rústicas sometidas a un proceso expropiatorio quizás sea el factor más determinante en la fijación del precio y el que 
más dificultades y controversia ha generado desde que el método de capitalización de rentas está vigente como criterio legal de valoraciones.

Como se ha comentado anteriormente, el tipo de interés se fija legalmente. Atendiendo al Reglamento de Valoraciones del RD 1492/2011 el tipo a aplicar se corresponde con el producto de una capitalización general (r1) coincidente con el rendimiento de las letras y bonos del Tesoro de dos a seis años y una capitalización correctora (r2) según el tipo de cultivo o aprovechamiento.

De esta manera, el tipo r1 asemeja la rentabilidad agraria como un coste de oportunidad en los mercados financieros y el tipo $\mathrm{r} 2$ modula la rentabilidad concreta del cultivo. No todos los cultivos arrojan la misma renta ni tienen la misma rentabilidad. Se podría determinar de manera general que cultivos con mayor renta de explotación, como los de regadío, cultivos altamente tecnificados como hortícolas en invernadero, etc...obtienen mayor rentabilidad frente a cultivos como por ejemplo cultivos extensivos en secano y los aprovechamientos de pastos cuya renta en menor y su rentabilidad más baja.

Como ejemplo, en la siguiente tabla se exponen los valores de r2 para los cultivos y aprovechamientos más significativos, recogido en el anexo 1 del RD 1492/2011.

Tabla 1. Coeficientes correctores del tipo de capitalización en explotaciones agropecuarias y forestales

\begin{tabular}{|l|c|}
\hline Cultivo o aprovechamiento & Coeficiente corrector \\
\hline Tierras labor secano & 0,49 \\
\hline Tierras labor regadío & 0,78 \\
\hline Cultivos protegidos regadío & 0,78 \\
\hline Frutales cítricos & 0,61 \\
\hline Viñedo & 0,59 \\
\hline Olivar & 0,43 \\
\hline Pastizales & 0,51 \\
\hline Explotaciones forestales & 0,58 \\
\hline
\end{tabular}

La tierra entendida como una inversión, con respecto a inversiones financieras, tiene unas particularidades que hacen imposible su analogía: su liquidez es baja, la seguridad es alta por lo que el factor riesgo es mínimo y su rentabilidad es muy variable. Esta rentabilidad agraria se encuentra entre el $2 \%$ y el $5 \%$. Fijando legalmente, por tanto, el tipo de capitalización y adecuándolo a tipos de interés financiero, no existe ninguna garantía de que el valor de capitalización que se obtiene como resultado sea un estimador fiable del valor real del bien o del valor de mercado.

De hecho, establecer como tipo de capitalización base el rendimiento interno mercados secundarios entre 2 y 6 años, dato que se publica mensualmente en el BOE, se antojaba una solución adecuada para reflejar de una manera más o menos objetiva la rentabilidad agraria puesto que se suele encontrar en el umbral del $4 \%$. Pero analizando la evolución del mercado de deuda que ha estado sujeta durante un periodo largo de tiempo a las tensiones propias del escenario de recesión económica y crisis financiera que hemos sufrido, este indicador ha experimentado un desplome constante. Si a esta bajada de los rendimientos de deuda, le añadimos la aplicación de los factores correctores del tipo en función del cultivo, ha llevado a un aumento significativo y desproporcionado del valor indemnizatorio del suelo rústico y un alejamiento desmesurado de los valores de mercado.

Para facilitar la comprensión de este fenómeno, que ha ocasionado verdaderos problemas administrativos y judiciales, puesto que los organismos expropiantes han estado obligados a ofrecer justiprecios en consonancia con el imperativo legal para su cálculo, se 
expone el siguiente caso práctico de evolución temporal del valor de mercado y del valor de expropiación en euros por hectárea de un explotación media y representativa del cultivo de algodón en regadío en el Valle del Guadalquivir, considerando la variación del tipo de capitalización en el periodo comprendido de su aplicación (2.007-2.015). Para su ejemplificación se ha tomado el valor de 650 €/ha como referencia de renta de la tierra y un factor de localización medio de 1,5.

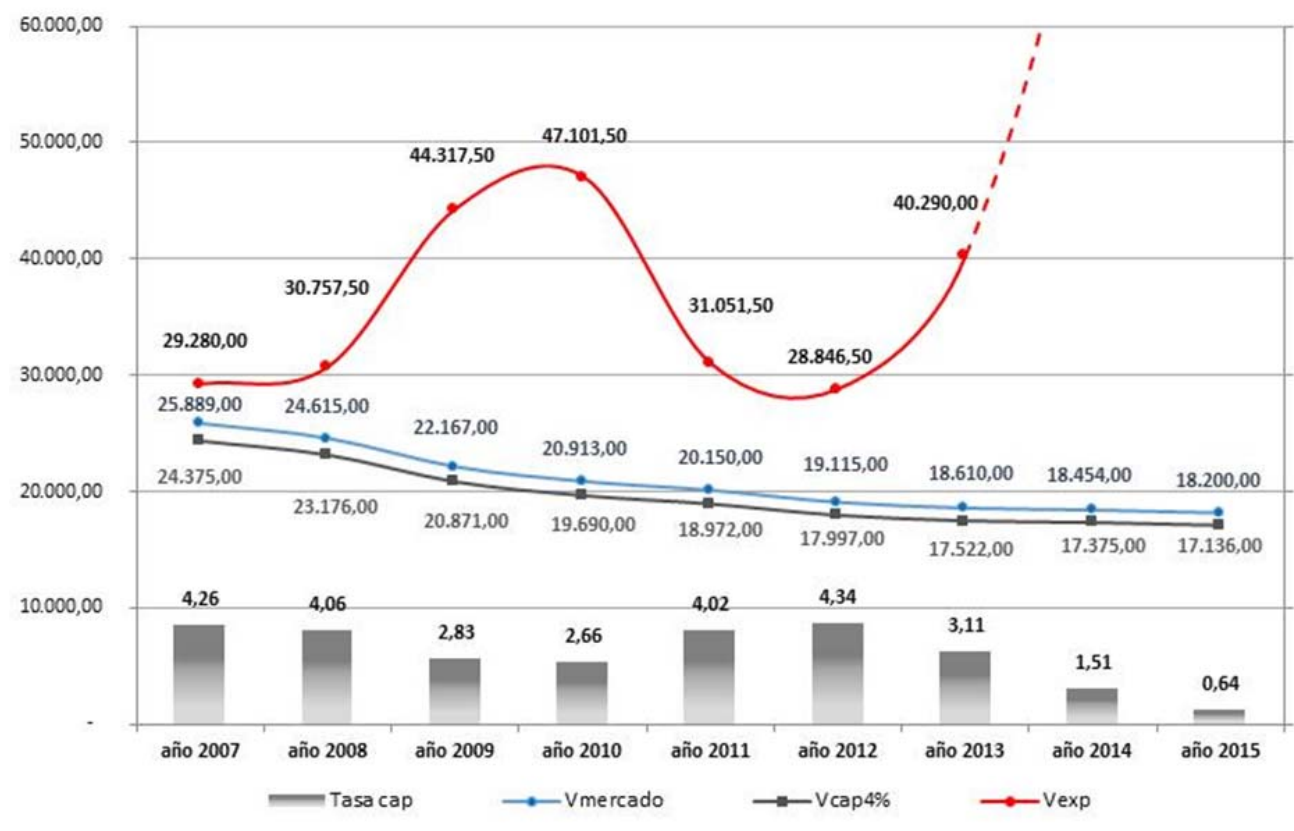

Figura 1. Evolución de los valores de la tierra en €/ha en cultivo de algodón en regadío en el Valle del Guadalquivir

Así pues, poniéndonos en el caso de una comunidad de regantes que tuviese que hacer frente a la expropiación de 16 hectáreas para la ejecución de balsas, de una primera estimación ajustándose a valor de mercado para el año 2013, que ronda los $300.000 €$, tendría que enfrentarse a una valor final de 650.000 € en la expropiación.

El efecto se agrava aún más en aquellos cultivos que tienen un factor de corrección más bajo, como por ejemplo labores de secano, en los que el tipo de capitalización a aplicar es la mitad del fijado anteriormente.

Ante esta situación en la que el tipo de interés dispara los precios de la tierra, se han sucedido diferentes mecanismos para minimizar su efecto. Los Jurados de Expropiación han tomado tipos medios anuales en lugar del mensual correspondiente para aumentar su valor; se han tomado valores de renta de la tierra más bajos e incluso se han tomado precios medios entre los valores estadísticos de mercado y valores analíticos calculados. Todo ello realizando interpretaciones de la norma contenida en el reglamento de valoraciones. Esto supone un conflicto jurídico puesto que esta situación favorece a los titulares afectados, comprometiendo muy gravemente la viabilidad del proyecto al no poder hacer frente al importe total de las expropiaciones del terreno por donde se va a ejecutar la obra prevista.

El primer intento de solución del problema ocasionado con el tipo de capitalización, se materializa en la Ley $37 / 2015$, de 29 de septiembre, de carreteras, que en su disposición final tercera, modificación del texto refundido de la Ley del suelo, estableciendo el tipo de capitalización como el valor promedio de los datos anuales publicados por el Banco de España de la rentabilidad de las Obligaciones del Estado a 30 años, correspondientes a los 
tres años anteriores a la fecha a la que deba entenderse referida la valoración. Este nuevo valor también se fija en el vigente el Texto refundido de la Ley de Suelo y Rehabilitación Urbana.

Esta modificación de la tasa o interés a aplicar soluciona, en parte, reflejando valores de capitalización más razonable; primero, porque la rentabilidad a largo plazo se comporta de una manera más estable y segundo, al tratarse de valores medios anuales establece, por lo menos, un comportamiento constante a lo largo de la tramitación del expediente expropiatorio. Pero, sin embargo, es un valor legal que sigue sometido a tensiones financieras sin ajustarse a la rentabilidad real agraria.

\subsubsection{Análisis de los justiprecios fijados por los Jurados de Expropiación.}

Los jurados de Expropiación, provinciales o territoriales, se han comportado de manera distinta ante esta situación como se ha dejado indicado anteriormente.

Por la propia naturaleza y composición de los Jurados, éstos pueden ponderar situaciones y establecer criterios, dentro de un ámbito legal común, que pueden no ser compartidos por las partes.

Como ejemplo y compendio de todo lo analizado en este trabajo, se expone el siguiente cuadro, en el cual se evalúan a lo largo de un periodo reciente de tiempo los justiprecios fijados por distintos Jurados provinciales de Castilla y León sobre actuaciones de modernización de regadíos análogas en zona territorial y precios medios para terrenos de labor regadío.

Tabla 2. Evolución de los Justiprecios fijados por Jurados de Expropiación para fincas de labor regadío en Castilla y león

\begin{tabular}{|l|l|c|c|}
\hline Jurado Provincial & \multicolumn{1}{|c|}{ Actuación } & \multicolumn{1}{|c|}{$\begin{array}{c}\text { Fecha de } \\
\text { valoración }\end{array}$} & $\begin{array}{c}\text { Justiprecio } \\
\text { Fijado } \\
\text { (f/ha) }\end{array}$ \\
\hline PALENCIA & CANAL PISUERGA & Abril 2008 & 17.200 \\
\hline VALLADOLID & CANAL DE RIAZA & Abril 2008 & 19.200 \\
\hline BURGOS & CANAL DE RIAZA & Mayo 2008 & 14.400 \\
\hline VALLADOLID & CANAL DE TORDESILLAS & Abril 2009 & 23.700 \\
\hline BURGOS & CANAL DE GUMA & Mayo 2010 & 22.200 \\
\hline ZAMORA & CANAL DE TORO & Septiembre 2012 & 34.100 \\
\hline ZAMORA & CANAL DE TORO SECTOR III & Octubre 2013 & 34.700 \\
\hline ZAMORA & CANAL DE TORO SECTOR IV & Octubre 2013 & 39.600 \\
\hline
\end{tabular}

Se puede observar claramente la tendencia alcista de precios fijados como reflejo de la realidad cambiante a efectos valorativos. También se distingue los diferentes comportamientos de Jurados provinciales distintos sobre una misma realidad a valorar como ocurre en abril de 2008 para los territorios de Valladolid y Burgos.

\section{4 - Conclusiones}

Después de haber analizado los factores legales, económicos y técnicos, a través de la legislación que ha estado vigente sobre valoración de terrenos a efectos expropiatorios, para un correcto dimensionamiento de la partida de gasto en la que tienen que incurrir los beneficiarios de obras de modernización de regadíos, se proponen las siguientes conclusiones: 
1. A nivel de proyecto de ejecución de obras de modernización de regadíos, el Anejo de expropiaciones debe ser una herramienta objetiva y eficaz para establecer la horquilla de precios probable del procedimiento expropiatorio ajustándose a legislación vigente y a la realidad valorativa.

2. Establecer jurídicamente un régimen de valoración en el ámbito de las expropiaciones en las que el método de fijación de los precios del suelo no esté distanciado significativamente del valor de mercado de los terrenos, proponiendo para ello figuras mixtas entre métodos comparativos y métodos analíticos.

3. Disponer de información objetiva y fiable de las zonas territoriales específicas de explotación en el medio rural, atendiendo cuidadosamente a la diversidad de cultivos, gestión de la tierra y parámetros técnico-económicos para establecer unos valores medios reales en los que se establezca un umbral de precios invitando a los servicios de prospectiva, análisis y estadística de los organismos competentes en materia de precios y rendimientos a crear una base de datos de explotaciones tipo incluso a nivel de comarca. Esta herramienta sería de gran utilidad y convergencia para todos los agentes que intervienen en un procedimiento expropiatorio, desde el propietario afectado a los Jurados de Expropiación

4. El tipo de capitalización a aplicar legalmente debe ser reflejo de la rentabilidad de los mercados agrarios sin estar sujeto a tensiones impropias y coyunturales de los ciclos de la economía.

5. Dado que muchas de las valoraciones pasan por los Jurados de Valoración, estos deberían de homogeneizar más los criterios evaluatorios. 